\title{
Increased regional cerebral glucose uptake in an APP/PS1 model of Alzheimer's disease
}

\author{
Géraldine Poisnel $^{\mathrm{a}, \mathrm{b}, \mathrm{c}, \mathrm{d}}$, Anne-Sophie Hérard ${ }^{\mathrm{b}, \mathrm{c}}$, Nadine El Tannir El Tayara ${ }^{\mathrm{e}, \mathrm{f}}$, \\ Emmanuel Bourrin ${ }^{\mathrm{b}, \mathrm{c}, \mathrm{d}}$, Andreas Volk ${ }^{\mathrm{e}, \mathrm{f}}$, Frank Kober ${ }^{\mathrm{g}}$, Benoit Delatour ${ }^{\mathrm{h}, \mathrm{i}, \mathrm{j}}$, \\ Thierry Delzescaux ${ }^{\mathrm{b}, \mathrm{c}}$, Thomas Debeir ${ }^{\mathrm{a}}$, Thomas Rooney ${ }^{\mathrm{a}}$, Jésus Benavides ${ }^{\mathrm{a}}$, \\ Philippe Hantraye ${ }^{\mathrm{b}, \mathrm{c}}$, Marc Dhenain ${ }^{\mathrm{b}, \mathrm{c}, *}$ \\ ${ }^{a}$ Therapeutic Strategic Unit Aging, Alzheimer/Parkinson/Stroke, Chilly-Mazarin, France \\ ${ }^{b}$ CEA, I2BM, MIRCen, Fontenay aux Roses, France \\ ${ }^{c}$ CNRS, URA 2210, Fontenay aux Roses, France \\ ${ }^{d}$ Servier Research Institute, Division of Neurodegenerative Diseases, Croissy-sur-Seine, France \\ ${ }^{e}$ INSERM, U759, Centre Universitaire, Laboratoire 112, Orsay Cedex, France \\ ${ }^{f}$ Institut Curie, Centre Universitaire, Laboratoire 112, Orsay Cedex, France \\ ${ }^{g}$ Centre de Résonance Magnétique Biologique et Médicale (CRMBM), UMR CNRS 6612, Faculté de Médecine, Université de la Méditerranée, \\ Marseille, France \\ ${ }^{h}$ CNRS, UMR 7225, Paris, France \\ ${ }^{i}$ INSERM, U975, Paris, France \\ ${ }^{j}$ Université Pierre \& Marie Curie-Paris6, Faculté de Médecine, UMR S975, CRICM, Paris, France
}

Received 3 March 2011; received in revised form 15 September 2011; accepted 16 September 2011

\begin{abstract}
Alzheimer's disease (AD), the most common age-related neurodegenerative disorder, is characterized by the invariant cerebral accumulation of $\beta$-amyloid peptide. This event occurs early in the disease process. In humans, [18F]-fluoro-2-deoxy-D-glucose ([18F]-FDG) positron emission tomography (PET) is largely used to follow-up in vivo cerebral glucose utilization (CGU) and brain metabolism modifications associated with the Alzheimer's disease pathology. Here, [18F]-FDG positron emission tomography was used to study age-related changes of cerebral glucose utilization under resting conditions in 3-, 6-, and 12-month-old $\mathrm{APP}_{\mathrm{SweLon}} / \mathrm{PS}_{\mathrm{M} 146 \mathrm{~L}}$, a mouse model of amyloidosis. We showed an age-dependent increase of glucose uptake in several brain regions of APP/PS1 mice but not in control animals and a higher [18F]-FDG uptake in the cortex and the hippocampus of 12-month-old APP/PS1 mice as compared with age-matched control mice. We then developed a method of 3-D microscopic autoradiography to evaluate glucose uptake at the level of amyloid plaques and showed an increased glucose uptake close to the plaques rather than in amyloid-free cerebral tissues. These data suggest a macroscopic and microscopic reorganization of glucose uptake in relation to cerebral amyloidosis.
\end{abstract}

(C) 2012 Elsevier Inc. All rights reserved.

Keywords: Alzheimer; Amyloid; APP/PS1 transgenic mice; Biomarker; Cerebral glucose uptake; [14C]-2-deoxyglucose; [18F]-FDG-PET

\footnotetext{
* Corresponding author at: MIRCen, CEA/CNRS URA 2210, 18 Route du Panorama, 92265 Fontenay aux Roses, France. Tel.: +33 14654 81 92; fax: +33146548451 .

E-mail address: marc.dhenain@cea.fr (M. Dhenain).
}

\section{Introduction}

Alzheimer's disease (AD), the most common agerelated neurodegenerative disorder, is a critical public health issue in our societies. Intracerebral amyloid- $\beta$ deposits in the form of amyloid plaques are strongly associated with the pathogenesis of this disease (Jellinger and Bancher, 1998). 
The discovery of new drugs against $\mathrm{AD}$ is largely based on the use of transgenic ( $\mathrm{tg}$ ) mouse models of amyloidosis expressing mutant forms of human amyloid precursor protein (APP) and presenilin-1 (PS1) (Duyckaerts et al., 2008). The use of these models in preclinical trials requires the validation of translational biomarkers. Ideally, such biomarker should rely on the same technology in humans and in animal models. In both species, such biomarkers should allow evaluating in vivo modifications of a biological endpoint after administration of an experimental drug. The changes of a translational biomarker should also be similar in animals and humans during pathology evolution or drug administration. If so, modifications of a biomarker in preclinical trials allow predicting expected changes in clinical trials.

Cerebral glucose metabolism, as measured by [18F]fluoro-2-deoxy-D-glucose ([18F]-FDG) positron emission tomography (PET), is one of the most sensitive functional biomarkers of AD (Herholz et al., 2007; Silverman et al., 2001). Indeed, AD patients display early and progressive reductions of glucose metabolism in cortical and hippocampal regions (Mosconi et al., 2005; Nestor et al., 2004).

Cerebral glucose utilization (CGU) has been mainly studied in tg AD mice using [14C]-2-deoxyglucose (2DG) or FDG autoradiography, a method that requires the euthanasia of the animals and does not allow longitudinal assessments. Results varied depending on brain structures, models, and age of evaluated animals. For example, reductions of CGU were observed in the cortex and hippocampus of 2-3-month-old amyloid deposit-free $\mathrm{APP}_{(\mathrm{Tg} 2576)}$ mice (Niwa et al., 2002). By contrast, an increase of CGU was described in cortical areas and hippocampus of 2-, 12(Valla et al., 2008), and 17-month-old PDAPP ${ }_{\mathrm{V} 717 \mathrm{~F}}$ mice, whereas a decrease of CGU was observed in some other brain structures such as the posterior cingulate cortex in 3-, 10-, and 17-month-old PDAPP $\mathrm{V}_{\mathrm{V} 17 \mathrm{~F}}$ mice (Reiman et al., 2000). In $\mathrm{APP}_{(\mathrm{Tg} 2576)} \mathrm{xPS} 1_{\mathrm{M} 146 \mathrm{~L}}$ mice, an increased CGU was detected in some cortical areas at 16 months of age (Valla et al., 2006).

Advances in [18F]-FDG-PET technology have now enabled the accurate in vivo evaluation of CGU in small animals (Matsumura et al., 2003). In mice, one major technical difficulty in measuring CGU is the need for arterial blood sampling which cannot be repeatedly done in the same animal without modifying profoundly its homeostasis. This is probably why only few studies have been performed in AD mouse models. After somatosensory activation, a reduction of cortical CGU increase was demonstrated in PDAPP $_{\text {SweLon }}$ mice (Nicolakakis et al., 2008). Two studies evaluated brain metabolism under resting conditions and did not depict any significant alteration in 10-15-month-old $\mathrm{APP} 23$ or $\mathrm{APP}_{(\mathrm{Tg} 2576)}$ mice (Heneka et al., 2006; Kuntner et al., 2009). A recent investigation highlighted brain hypermetabolism in 7- and 19-month-old $\mathrm{APP}_{(\mathrm{Tg} 2576)}$ mice (Luo et al., 2010). These mice start to develop amyloid deposits at 9 to 12 months (Hsiao et al., 1996). Some tg mouse models develop a more severe phenotype and display amyloid plaques earlier. For example, in the $\mathrm{APP}_{751 \mathrm{SL}}$ $\mathrm{xPS}_{\mathrm{M} 146 \mathrm{~L}}(\mathrm{APP} / \mathrm{PS} 1)$ mice, extracellular amyloid deposition starts at the age of 2.5 months and then gradually increases (Blanchard et al., 2003; El Tannir El Tayara et al., 2006). The aim of our work was to evaluate, by in vivo [18F]-FDG-PET, the age-related changes of CGU under resting conditions in this double tg APP/PS1 mouse model. We focused on 3-, 6-, and 12-month-old APP/PS1 mice, as this is the age range used in pharmacological studies. We show that, compared with age-matched controls, APP/PS1 mice have a decreased glucose uptake at 3 months in the cortex and hippocampus as well as an increased glucose uptake in the cortex at 6 and 12 months and in the hippocampus at 12 months. The increased CGU in aged APP/ PS1 mice could be caused by a higher cerebral perfusion. We ruled out this hypothesis by showing, by in vivo magnetic resonance imaging (MRI) experiments, that perfusion is either constant or reduced in brain regions of aged APP/ PS1 mice. Finally, we developed a new technical approach based on 2DG-autoradiography to evaluate CGU within amyloid plaques and amyloid-free brain parenchyma. Thanks to this method, we showed an increased glucose uptake at the level of amyloid plaques rather than in adjacent amyloid-free cerebral parenchyma.

\section{Methods}

\subsection{Animals}

Animal experiment procedures were performed in strict accordance with the recommendations of the EEC (86/609/ EEC) and the French National Committee (decree 87/848) for the care and use of laboratory animals.

Our studies were performed in $\mathrm{APP}_{751 \mathrm{SL}} \mathrm{xPS} 1_{\mathrm{M} 146 \mathrm{~L}}$ (APP/PS1), PS1 $1_{\mathrm{M} 146 \mathrm{~L}}$, and C57/B16 mice. Heterozygous $\mathrm{APP}_{751 \mathrm{SL}} \mathrm{xPS} 1_{\mathrm{M} 146 \mathrm{~L}}$ double-tg mice were generated by crossing heterozygous tg $\mathrm{APP}_{(+/-)}$mice (Thy-1 $\mathrm{APP}_{751 \mathrm{SL}}$ [Swedish mutation KM670/671NL], London mutation V717I introduced in the human $\mathrm{APP}_{751}$ sequence) with homozygous tg $\mathrm{PS} 1_{(+/+)}$mice $\left(\mathrm{HMG} \mathrm{PS} 1_{\mathrm{M} 146 \mathrm{~L}}\right)$. The mice had been backcrossed on a C57/B16 background for more than 6 generations. Previous studies on these mice have shown that they do not exhibit cortical atrophy or corpus callosum agenesis (Delatour et al., 2006). $\mathrm{APP}_{(-/-)} /$ $\mathrm{PS}_{(+/-)}$mouse littermates ( $\mathrm{PS} 1_{\mathrm{M} 146 \mathrm{~L}}$ ) and $\mathrm{C} 57 / \mathrm{B} 16$ mice that do not display amyloid plaques were used as controls. Animals were housed at constant temperature $\left(22{ }^{\circ} \mathrm{C}\right)$ and humidity $(50 \%)$ with a 12-hour light/dark cycle.

\subsection{In vivo PET studies}

PET studies were performed in 3-, 6-, and 12-month-old $\mathrm{APP}_{751 \mathrm{SL}} \mathrm{xPS} 1_{\mathrm{M} 146 \mathrm{~L}}$ (APP/PS1), $\mathrm{PS}_{\mathrm{M} 146 \mathrm{~L}}$, and $\mathrm{C} 57 / \mathrm{B} 16$ mice ( $n=4$ females per group). The PET protocol was the following: animals were anesthetized by isoflurane (3\% for 
induction, $1 \%-1.5 \%$ for maintenance), and the respiration rate monitored during the experiment. The body temperature of the animals was maintained at $37{ }^{\circ} \mathrm{C}$ throughout the PET examination using a warming system.

PET images were recorded on a high-resolution smallanimal PET imaging device with a spatial resolution of 1.35 $\mathrm{mm}$ and a field of view (FOV) of $7.6 \mathrm{~cm}$ (MicroPET Focus220, Siemens Medical Solutions, Inc., Hoffman Estates, IL, USA) (Tai et al., 2005). The mice were scanned with an energy window of 350-650 keV and a coincidence time window of 6 nseconds. Brain emission scans were acquired in 3-D mode during 60 minutes after a tail-vein bolus injection of $16.8 \pm 0.4$ MBq of [18F]-FDG (CisBio, Orsay, France).

Blood glucose concentration was measured once during the scan using a One Touch Ultra glucose meter (LifeScan, Issy-Les-Moulineaux, France). Blood glucose concentrations were in the normal range (Yu et al., 2009) (APP/PS1 3 months, $10.8 \pm 1.2 \mathrm{mmol} / \mathrm{L} ; 6$ months, $10.9 \pm 0.8$ $\mathrm{mmol} / \mathrm{L} ; 12$ months, $13.2 \pm 1.9 \mathrm{mmol} / \mathrm{L}$; PS1 3 months, $11.9 \pm 0.8 \mathrm{mmol} / \mathrm{L} ; 6$ months, $8.2 \pm 0.9 \mathrm{mmol} / \mathrm{L} ; 12$ months, $11.1 \pm 0.9 \mathrm{mmol} / \mathrm{L}$; C57B16 3 months, $10.9 \pm 0.8$ $\mathrm{mmol} / \mathrm{L} ; 6$ months, $11.6 \pm 2.5 \mathrm{mmol} / \mathrm{L} ; 12$ months, $11.1 \pm$ $1.9 \mathrm{mmol} / \mathrm{L}$ ) and no difference was detected between the various genotypes or ages of the groups (2-way analysis of variance [ANOVA] with genotype and age as betweensubject factors, $F \mathrm{~s}<1$ for the main factors).

The PET images were reconstructed with the 2-D iterative ordered-subset expectation maximization (FORE 2D OSEM) mode. Sixteen subsets and 4 iterations were used for reconstruction. The voxel size was $0.5 \times 0.5 \times 0.8$ $\mathrm{mm}^{3}$. The reconstructed images were examined with a 3-D display in axial, coronal, and sagittal views.

Cerebral glucose uptake was evaluated in 4 volumes of interest (VOI), consisting of the cortex (from bregma 1.3 to -3.5 according to Paxinos and Franklin, 2001), the hippocampus (from bregma 1.2 to -3.0 ), the striatum (from bregma 1.0 to -0.9 ), and the cerebellum (from bregma -6.3 to -7.5$)$. The VOIs used in the current study were larger than $9 \mathrm{~mm}^{3}$, as a recent study on a microPET system similar to ours (Constantinescu and Mukherjee, 2009) showed that this size avoids partial volume effects (Phelps, 2006). Also, the VOIs were far from harderian glands to avoid spill-over effects. To ensure proper VOI placement on PET images, we coregistered microPET images with T2-weighted MRI scans from a representative mouse from each experimental group. MR images were recorded on a 4.7 Tesla Bruker Biospec 47/30 system, equipped with a $12 \mathrm{~cm}$ diameter gradient system $(200 \mathrm{mT} / \mathrm{m})$. A surface coil (diameter $=30 \mathrm{~mm}$ ), actively decoupled from the transmitting birdcage probe (Bruker BioSpin SAS, Wissembourg, France) was used for signal acquisition. Three-dimensional fast spin-echo (RARE) images were recorded with an isotropic nominal resolution of $117 \mu \mathrm{m}$ (repetition time/echo time $[\mathrm{TR} / \mathrm{TE}] /$ weighted-TE $=$ $2500 / 10 / 75.4 \mathrm{msec}$, RARE factor $=16$, number of averages
[NA] $=1$, field of view $=3 \times 1.5 \times 1.5 \mathrm{~cm}$, matrix $=$ $256 \times 128 \times 128$ ) (Delatour et al., 2006).

The mean $[18 \mathrm{~F}]-\mathrm{FDG}$ activities, corrected for radioactive decay, were evaluated for each VOI on integrated PET images recorded during a 30-60-minute acquisition period. Standardized uptake values (SUV) were obtained for each VOI by dividing the mean $[18 \mathrm{~F}]-\mathrm{FDG}$ activities by the injected dose and the animal weight. Then, regional FDG data were normalized by the FDG uptake within the cerebellum, a structure devoid of amyloid plaques in the APP/PS1 mice (Blanchard et al., 2003).

\subsection{MRI-based evaluation of cerebral perfusion}

Cerebral perfusion was evaluated in 12-month-old APP/ PS1 and PS1 mice ( $n=9$ and 10, respectively). Cerebral blood flow (CBF) was evaluated by using a pulsed arterial spin labeling technique. The animals were anesthetized with isoflurane (3\% for induction, 1\%-1.5\% for maintenance), as previously described (Faure et al., 2011). In vivo MRI images were recorded on a 4.7 Tesla Biospec 47/30 system (Bruker) with the same set-up used for the T2-weighted images. Perfusion measurements were performed using a flow sensitive alternating inversion recovery (FAIR) LookLocker gradient-echo method (Kober et al., 2008). Briefly, a series of 50 echoes were acquired after global or sliceselective magnetization inversion with the following imaging parameters: TE/TR $=1.59 / 150 \mathrm{~ms}, \alpha=12.5^{\circ}$, field of view: $20 \times 20 \mathrm{~mm}^{2}$, slice thickness: $1.5 \mathrm{~mm}$, input matrix: $128 \times 64$; total acquisition time 32 minutes. Data acquisition was performed in a tissue slab of the posterior cerebrum (volume acquisition: from bregma -1.82 to bregma -3.28 according to Paxinos and Franklin, 2001). Because the thickness of this sampling slice was too large to measure perfusion in convoluted VOIs, such as the hippocampus, we evaluated perfusion in large cortical and thalamic VOIs with minimal shape variations across the anteroposterior axis of the studied slice. In these selected VOIs perfusion values (P), expressed as $\mathrm{mL} \cdot \mathrm{g}^{-1} \mathrm{~min}^{-1}$, were calculated as follows: $\mathrm{P} / \lambda=[(\mathrm{T} 1$ global $/ \mathrm{T} 1$ blood $) \times(1 / \mathrm{T} 1$ selective $-1 /$ T1global)] (program developed in an IDL environment; IDL 7.1, RSI, Boulder, CO, USA). T1 global was calculated from images recorded with a global inversion pulse while T1 selective was calculated with the selective inversion pulse. The blood/tissue partition coefficient for water $(\lambda)$ was assumed to be $0.9 \mathrm{~mL} / \mathrm{g}$ (Sun et al., 2004), and T1 blood was 1.7 seconds (Williams et al., 1992).

\subsection{Microscopic analysis of glucose utilization}

In order to better interpret at the cellular level, the increase in glucose uptake observed by PET, we performed a complementary autoradiographic study using 2DG and a new image processing protocol (Dubois et al., 2007). This method allows to perfectly register autoradiographic and histological sections. After definition of microscopic regions of interest (ROIs) on histological sections, one can 
paste the ROIs on autoradiograms, and evaluate the CGU within these microscopic regions.

Four APP/PS1 (12-month-old) and 3 age-matched control PS1 mice were evaluated. Animals were anesthetized with isoflurane and a catheter was inserted intraperitoneally. Mice were then allowed to recover from anesthesia for 1 hour until 2DG injection $(16.5 \mu \mathrm{Ci} / 100$ g body weight; PerkinElmer, Boston, MA, USA). After a 45-minute uptake period, the animals were euthanized by injection of a lethal dose of sodium pentobarbital. The brain was removed and immediately snap-frozen in isopentane at $-40{ }^{\circ} \mathrm{C}$ before being entirely cut on a CM3050S cryostat (Leica, Rueil-Malmaison, France) into $20-\mu \mathrm{m}$ thick coronal sections. One out of 4 sections were mounted on Superfrost (Menzel-glaser, GmbH, Braunsheig, Germany) glass slides, heat-dried, and exposed onto the autoradiographic film (Kodak Biomax MR, Sigma-Aldrich, France) for 1 week, together with radioactive [14C] standards (146C, American Radiochemical Company, St. Louis, MO, USA). The same sections were then processed for Nissl staining to provide complementary information about brain anatomy and amyloid load. The autoradiograms, the corresponding Nissl stained sections, and the [14C] standards were digitized as 8-bit gray-scale images, using a flatbed scanner (ImageScanner, GE Healthcare, Orsay, France) with a 1200 dpi (dots per inch) in-plane resolution (pixel size $21 \times 21 \mu^{2}$ ), as previously described (Dubois et al., 2007). Each 2-D autoradiographic section was coregistered with its corresponding histological Nissl section.

Several ROIs of $42 \times 42 \mu \mathrm{m}^{2}$, corresponding to regions containing amyloid plaques, were outlined in the cortex ( $n=100$ different ROIs; 25 per animal), hippocampus ( $n=36$ different ROIs; 9 per animal), and striatum ( $n=36$ different ROIs; 9 per animal) from histological sections from APP/PS1 mice. The same number of ROIs were positioned over regions without amyloid plaques in APP/PS1 and in control PS1 mice. The ROIs were then pasted on corresponding registered autoradiograms and the radioactivity counted. Radioactivity was also assessed from the cerebellum of the animals. A glucose utilization index was obtained for each ROI by dividing the regional radioactivity values by the radioactivity within the cerebellum. Data were evaluated as a percentage of radioactivity compared with radioactivity values in the amyloid-free regions of PS1 mice.

\subsection{Immunohistochemistry and histochemistry}

Further histopathological analysis was performed on the mice used in the PET studies. Immediately after the PET examination, animals were euthanized by an overdose of $5 \%$ isoflurane followed by cervical dislocation ( $n=9$ animals; 1 mouse per genotype and age group). The brains were quickly removed from the skull and immediately frozen in dry ice precooled isopentane and stored at $-20{ }^{\circ} \mathrm{C}$.
Then serial sagittal sections $(20 \mu \mathrm{m})$ were cut from frozen tissue using a cryostat and transferred to glass slides (Superfrost plus; Menzel-glaser, $\mathrm{GmbH}$ ). Prior to immunostaining, brain sections were fixed with $4 \%$ paraformaldehyde for 30 minutes at room temperature. Immunohistochemical detection of amyloidosis (4G8 antibody), reactive astrocytes (glial fibrillary acidic protein; GFAP), and microgliosis (Iba1) were performed using the Discovery XT automated stainer (Ventana, Strasbourg, France).

Sections for $\beta$-amyloid $(\mathrm{A} \beta)$ detection were pretreated with $80 \%$ formic acid for 3 minutes at room temperature and were then treated with a primary antibody (biotinylated mouse monoclonal antibody 4G8, 1/500; Covance Signet antibodies, Dedham, MA, USA) during 60 minutes at room temperature.

For reactive astrocyte detection, the slides were pretreated by an automated heat-induced epitope retrieval procedure (CC2 standard protocol, Ventana) and then processed with a rabbit polyclonal anti-GFAP; 1:1000 (Dako, Carpintera, CA, USA) during 60 minutes at room temperature.

For reactive microgliosis detection, the slides were pretreated by an automated heat-induced epitope retrieval procedure ( $\mathrm{CC} 1$ mild protocol; Ventana) and were then processed with an anti-Iba1 primary antibody (Iba1, 1/250; Wako Pure Chemicals, Richmond, VA, USA) during 60 minutes at room temperature.

For each staining, 32-minute incubation with a secondary antibody (biotinylated goat anti-rabbit IgG; 1/500; Vector Laboratories, Burlingame, CA, USA) was performed using the $\mathrm{ABC}$ method with diaminobenzidine (DAB Map kit, Ventana) as the chromogen (brown stain). Endogenous peroxidase activity and nonspecific binding sites were blocked according to the manufacturer's instructions. Endogenous biotin activity was blocked by adding an avidin/biotin blocking kit (Ventana) for 8 minutes.

The sections were then counterstained with hematoxylin (4 minutes; Ventana, 760-2021) and a bluing reagent (Ventana; 760-2037) was applied for counterstaining (4 minutes).

Amyloid was also labeled using Congo red staining (30 minutes in an $80 \%$ ethanol solution saturated with Congo red and sodium chloride, followed by rinsing in water, dehydration in ethanol, and clarification into toluene).

All slides were covered and evaluated by using a standard microscope (Olympus, Hamburg, Germany).

\subsection{Statistical analyses}

Statistical analyses of FDG and perfusion data were based on Pearson's and Mann-Whitney's tests. Autoradiographic data were analyzed using a 2-way ANOVA with animal and tissue type (location of the pixel within or outside amyloid deposits) as coefficients. Post hoc analyses were performed to analyze differences between glucose utilization index within amyloid plaques and within tissue without amyloid plaques. Statistica 7.1 (StatSoft, Inc., 


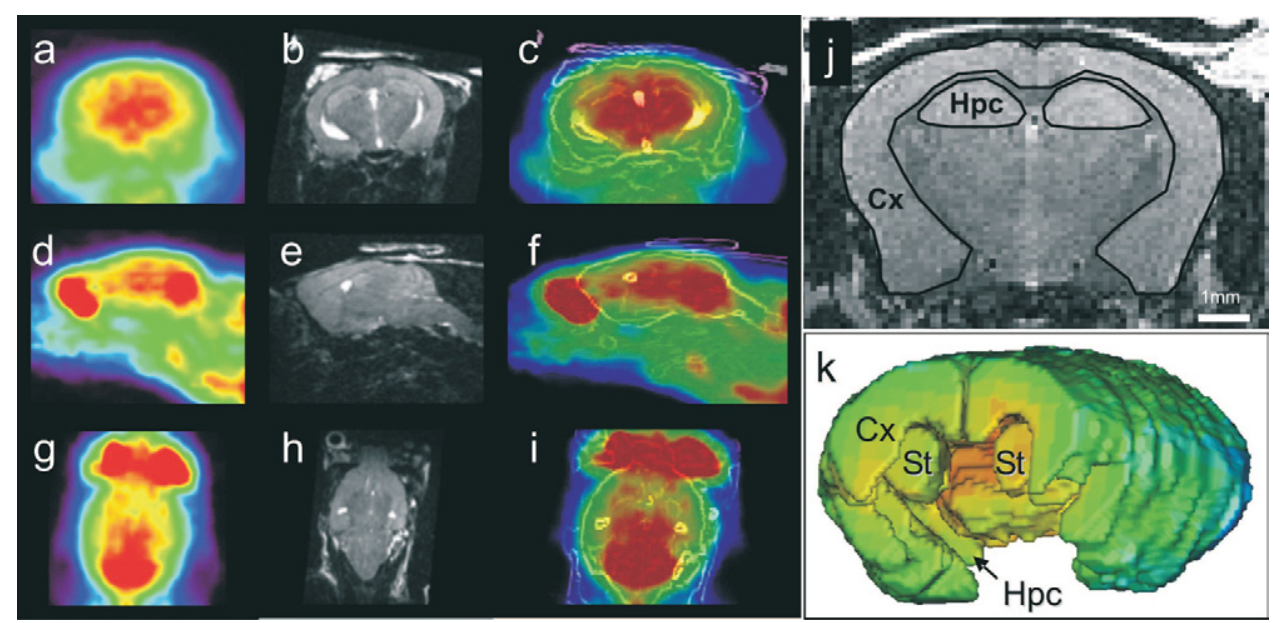

Fig. 1. [18F]-fluoro-2-deoxy-D-glucose ([18F]-FDG) positron emission tomography (PET) images from a 12-month-old APP/PS1 mouse in the coronal (a), sagittal (d), and horizontal (g) directions. Magnetic resonance (MR) images of the corresponding animal are shown in (b), (e), and (h). Registered PET and brain structure contours extracted from MR images are shown in (c), (f), and (i). Examples of regions of interest outlined on a MR imaging slice are shown in (j). Regions of interest were outlined on several slices from the whole brain. Three-D volumes showing the regions of interest are displayed in (k). Cx, cortex; Hpc, hippocampus; St, striatum.

Tulsa, OK, USA) was used for all the analysis. Two-tailed $p<0.05$ was considered statistically significant.

\section{Results}

\section{1. [18F]-FDG-PET study}

To study age-related changes of CGU, [18F]-FDG-PET images were recorded in APP/PS1, PS1, and C57/B16 mice at 3,6 , and 12 months of age. After anatomofunctional combination, cerebral regions, such as the cortex, the hippocampus, the striatum, and the cerebellum could be outlined on PET images (Fig. 1).

A significant positive correlation was found between the age of APP/PS1 mice and the [18F]-FDG uptake in the cortex $(r=0.87, p<0.001$; Fig. 2a), the hippocampus $(r=$ $0.76, p<0.005$; Fig. 2b), and the striatum $(r=0.60, p<$ 0.05; Fig. 2c). In control mice (either C57/B16 or PS1 mice), no such relationship between age and [18F]-FDG uptake could be seen, whatever the examined brain region $(p>$ 0.05; Fig. 2).

Evaluation of age-matched animals revealed a decrease of cortical and hippocampal glucose uptake in 3-month-old APP/PS1 mice $(0.8365 \pm 0.0099 ; 0.9660 \pm 0.0096$, respectively) as compared with PS1 mice (0.8647 \pm 0.0041 ; $1.0112 \pm 0.0055$, respectively) (Mann-Whitney test, $p<$ 0.05 ; Fig. 3a and b). At 6 months, the glucose uptake was increased in the cortex of APP/PS1 mice (0.9216 \pm 0.0309$)$ as compared with age-matched C57/B16 animals $(0.7637 \pm$ 0.0295; $p<0.05$; Fig. 3a). In 12-month-old APP/PS1 animals, the cortical glucose uptake was increased (1.0407 \pm $0.0343)$ as compared with PS1 $(0.8755 \pm 0.0345)$ and C57/B16 mice $(0.9095 \pm 0.0117 ; p<0.05$; Fig. 3a). An increase of glucose uptake was also detected in the hippocampus of 12-month-old APP/PS1 mice (1.2094 \pm
0.0602) as compared with age-matched C57/B16 animals (1.0340 $\pm 0.0067 ; p<0.05$; Fig. 3b).

\subsection{Cerebral perfusion}

To further evaluate the origin of the increased glucose uptake in aged APP/PS1 mice, we focused on another cohort of 12-month-old animals. First thanks to a pulsed arterial spin labeling technique, a cortical hypoperfusion was detected in the APP/PS1 mice ( $p<0.05$; Fig. 4 ). No change in cerebral blood flow was detected in the thalamus of the APP/PS1 mice ( $p>0.05$; Fig. 4 ).

\subsection{Microscopic analysis of glucose utilization}

A subgroup of the mice evaluated by spin labeling technique was further studied by autoradiography to evaluate CGU at the level of cortical, hippocampal, and striatal amyloid plaques. First, high-resolution autoradiograms were registered on Nissl-stained sections thanks to a spatial normalization algorithm. Nissl-stained sections were used to localize ROIs $\left(42 \times 42 \mu \mathrm{m}^{2}\right)$ either containing amyloid plaques or without amyloid plaques in APP/PS1 mice (Fig. 5a, c, e, and f) and amyloid free-ROIs in PS1 mice (Fig. 5b and d). The ROIs were then pasted on the corresponding autoradiographic sections from the same slices (Fig. 5e and f). A glucose utilization index was quantified in each ROI. In the cortex and the hippocampus, glucose uptake was higher in regions containing amyloid plaques compared with amyloid-free brain areas from APP/PS1 or PS1 mice (ANOVA followed by post hoc analysis, all $p<0.0001$; Fig. $5 \mathrm{~g}$ ).

\subsection{Neuropathological study}

As we have previously shown (El Tannir El Tayara et al., 2006), amyloid load detected by 4G8 immunohisto- 

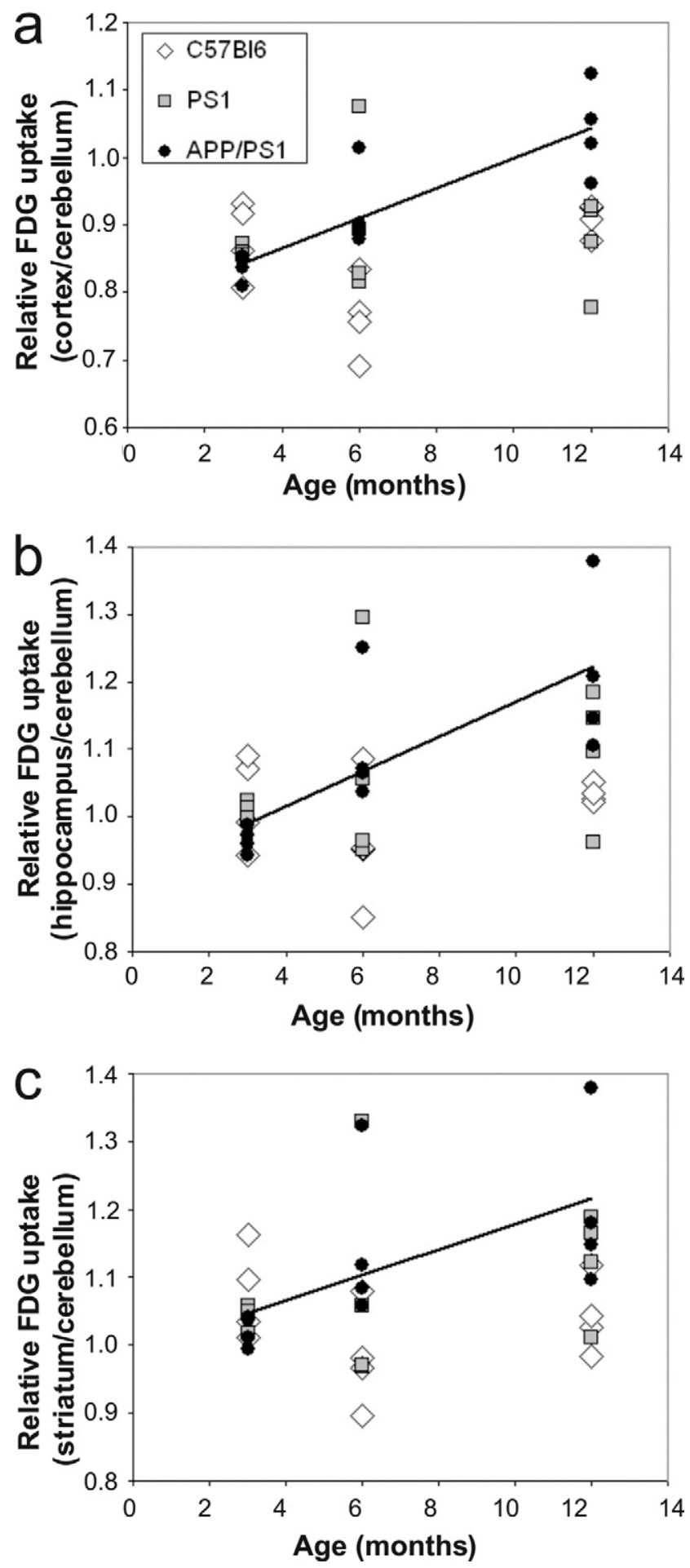

Fig. 2. Correlation between [18F]-fluoro-2-deoxy-D-glucose ([18F]-FDG) uptake and age in the cortex (a), hippocampus (b), and striatum (c) of C57/B16, PS1, and APP/PS1 mice. A significant correlation was found only in APP/PS1 mice (drawn lines).

chemistry and Congo red staining increased with age in APP/PS1 animals (Fig. 6): at 3 months of age APP/PS1 mice had sparse, but detectable, $\mathrm{A} \beta$ deposits and by 12 months of age, $A \beta$ deposits were distributed throughout the cortex and the hippocampus. In the striatum, amyloid plaques were rare and immature. No amyloid plaques were detected, at any age, either in PS1 or C57/B16 mice (Fig. 6).

Similarly, astrocytosis (GFAP; Fig. 6f-h) and microgliosis (Iba1 immunohistochemistry; Fig. 6j-i) increased with age in the cortex and the hippocampus of APP/PS1 mice: in 3 -month-old mice, astrocytes and microglia were evenly distributed throughout these regions. In old mice, intensely stained astrocytes and microglia were clustered around the amyloid plaques. As for amyloid plaques, astrocytosis, and
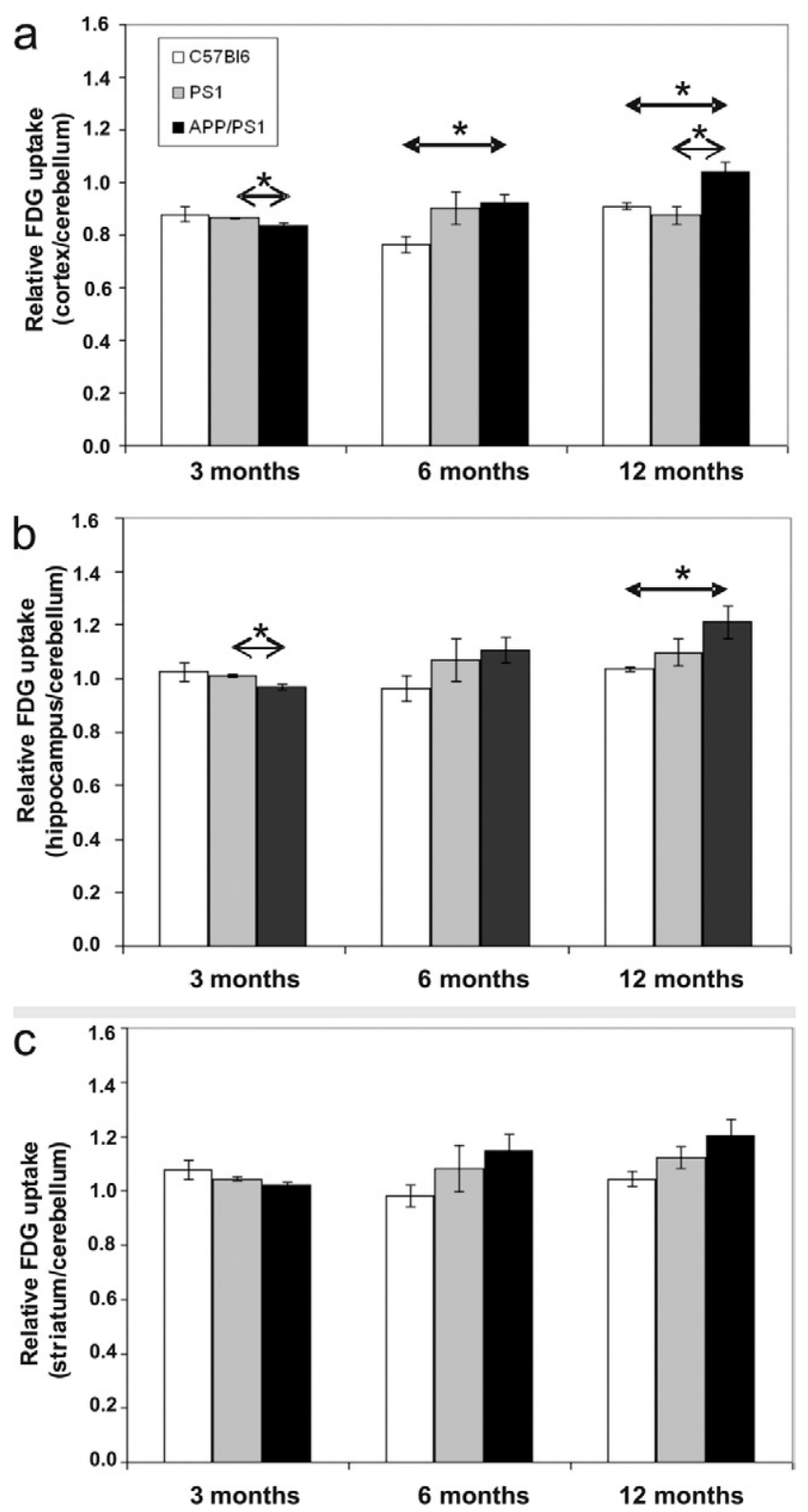

Fig. 3. Quantification of [18F]-fluoro-2-deoxy-D-glucose ([18F]-FDG) uptake in the cortex (a), hippocampus (b), and striatum (c) of C57/B16, PS1, and APP/PS1 mice at 3,6, and 12 months $(n=4$ per group; mean \pm standard error of the mean). Age-matched animals were compared by using the Mann-Whitney test $(* p<0.05)$. 


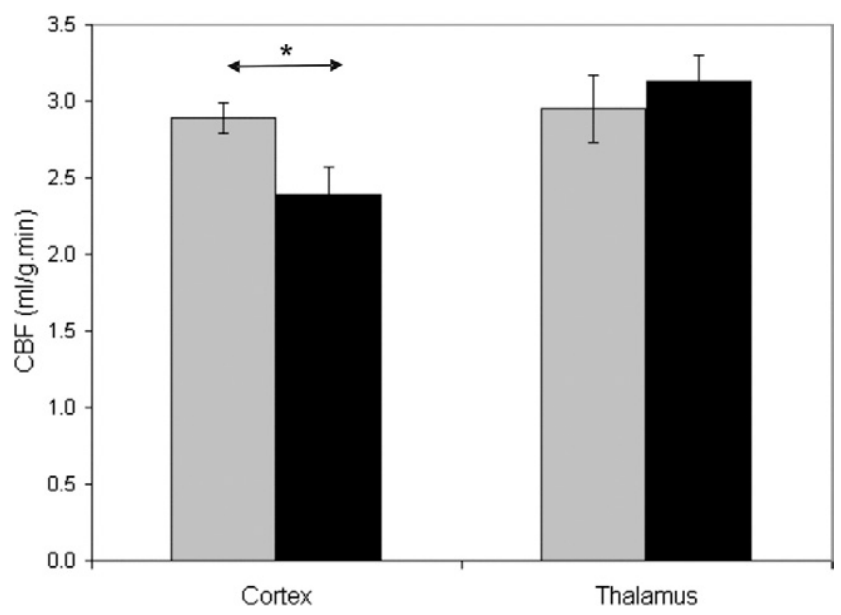

Fig. 4. Cerebral perfusion in the cortex and thalamus of 12-month-old APP/PS1 mice (black boxes) compared with age-matched PS1 animals (gray boxes) $(n=9-10$ per group; Mann-Whitney tests; $* p<0.05)$.

microgliosis, labeling was low in the striatum (Fig. 5h and i). As expected, GFAP and Iba1 immunoreactivities were low in the PS1 and C57/B16 mice (Fig. 6e and i).

\section{Discussion}

\subsection{Age-related increase of glucose uptake in APP/PS1 mice}

$[18 \mathrm{~F}]-\mathrm{FDG}$ is one of the most frequently used ligands for clinical PET imaging of cerebral metabolism in AD patients. Here, we have performed in vivo PET imaging to measure [18F]-FDG uptake in 3-, 6-, and 12-month-old APP/PS1 mice. This age range is the one typically used in pharmacological studies on this model.

In APP/PS1 mice, we observed an age-related increase of [18F]-FDG uptake in the cortex, the hippocampus, and the striatum. No age-related change in [18F]-FDG uptakes were detected in control PS1 and C57/B16 animals. This agerelated increase led to a higher [18F]-FDG uptake in the cortex and hippocampus of 12-month-old APP/PS1 mice as compared with age-matched PS1 or C57/B16 control mice. By contrast, in young 3-month-old APP/PS1 mice, there was a decrease of [18F]-FDG uptake in the cortex and the hippocampus. The discrepancy that we reported between young and old animals is consistent with results from the literature showing, at the cerebral and cellular levels, a hyperactivity in old (8-month) but not in young (2-month), amyloid-free, mouse brains (Busche et al., 2008; Kuchibhotla et al., 2009).

The age-related increase of cerebral metabolism in APP/ PS1 mice is however surprising because mouse models of AD show cognitive impairment and high amyloid deposition. Most of the studies, that have previously evaluated glucose uptake in the brain of AD mouse models, have used [14C]-FDG or [14C]-2DG autoradiography. Our results can be compared with these studies because regional CGU eval- uated by [18F]-FDG PET and [14C]-2DG autoradiography are strongly correlated (Toyama et al., 2004). Some of these studies have shown reduced brain metabolism in AD mouse models, but severe morphological alterations, such as agenesis of the corpus callosum, were detected in some of these models, e.g., the PDAPP ${ }_{\mathrm{V} 717 \mathrm{~F}}$ mice. These alterations could, in part, explain the decreased cerebral metabolism (Dodart et al., 1999; Gonzalez-Lima et al., 2001; Reiman et al., 2000). Several studies have also shown enhanced cerebral metabolism at more than 12 months in the cortex, the hippocampus, and the striatum of tg mouse models of $\mathrm{AD}$ (see studies in PDAPP ${ }_{\mathrm{V} 717 \mathrm{~F}}$ mice; Reiman et al., 2000; Valla et al., 2008; or $\mathrm{APP}_{(\mathrm{Tg} 2576)} / \mathrm{PS}_{\mathrm{M} 146 \mathrm{~L}}$ mice; Valla et al., 2006). A recent study by [18F]-FDG-PET also showed similar results in $\mathrm{APP}_{(\mathrm{Tg} 2576)}$ mouse model (Luo et al., 2010). These findings are consistent with our data in aged APP/PS1 mice. In addition to previous findings, our results demonstrate that glucose uptake increases progressively with aging in APP/PS1 mice. More importantly, our results are based on PET imaging. Unlike autoradiography, PET is a noninvasive method that allows following-up the animals for example during drug evaluations.

\subsection{FDG-PET in APP/PS1 mice: a translational biomarker?}

The increased glucose uptake that we observed in aged APP/PS1 mice is clearly different from the decreased glucose uptake described in syndromal AD patients (Mosconi, 2005). In these patients, the decreased [18F]-FDG uptake are supposed to be related to the presence of neurofibrillary tangles rather than amyloid deposition (Barrio et al., 2008; Choo et al., 2007; Edison et al., 2007). This hypothesis is supported by results obtained in a triple tg model of AD, which overexpresses human APP, PS1, and tau mutations. In these mice, the tau overexpression leads to a global age-related reduction of glucose uptake (Nicholson et al., 2010).

Our study, in an animal model of pure amyloidosis, thus emphasizes that amyloid deposits lead to an increased glucose uptake. Interestingly, in prodromal stages of $\mathrm{AD}$, an increased cerebral metabolism (Cohen et al., 2009) or a reorganization of brain metabolism (Vlassenko et al., 2010) have recently been described. Recent reviews suggest that tg mice simulate the asymptomatic phase of the disease rather than the clinical stage of the disease (Zahs and Ashe, 2010). The increased glucose uptake seen in tg mice is consistent with this view.

\subsection{Potential biological endpoints associated to increased glucose uptake in APP/PS1 mice}

Several cerebral alterations possibly occurring in tg mice could explain the increased glucose uptake in aged animals. These modifications could occur at the level of the vascular system or at the level of the tissue. Here, we evaluated 2 of them. First, alterations of the vascular function could lead to 


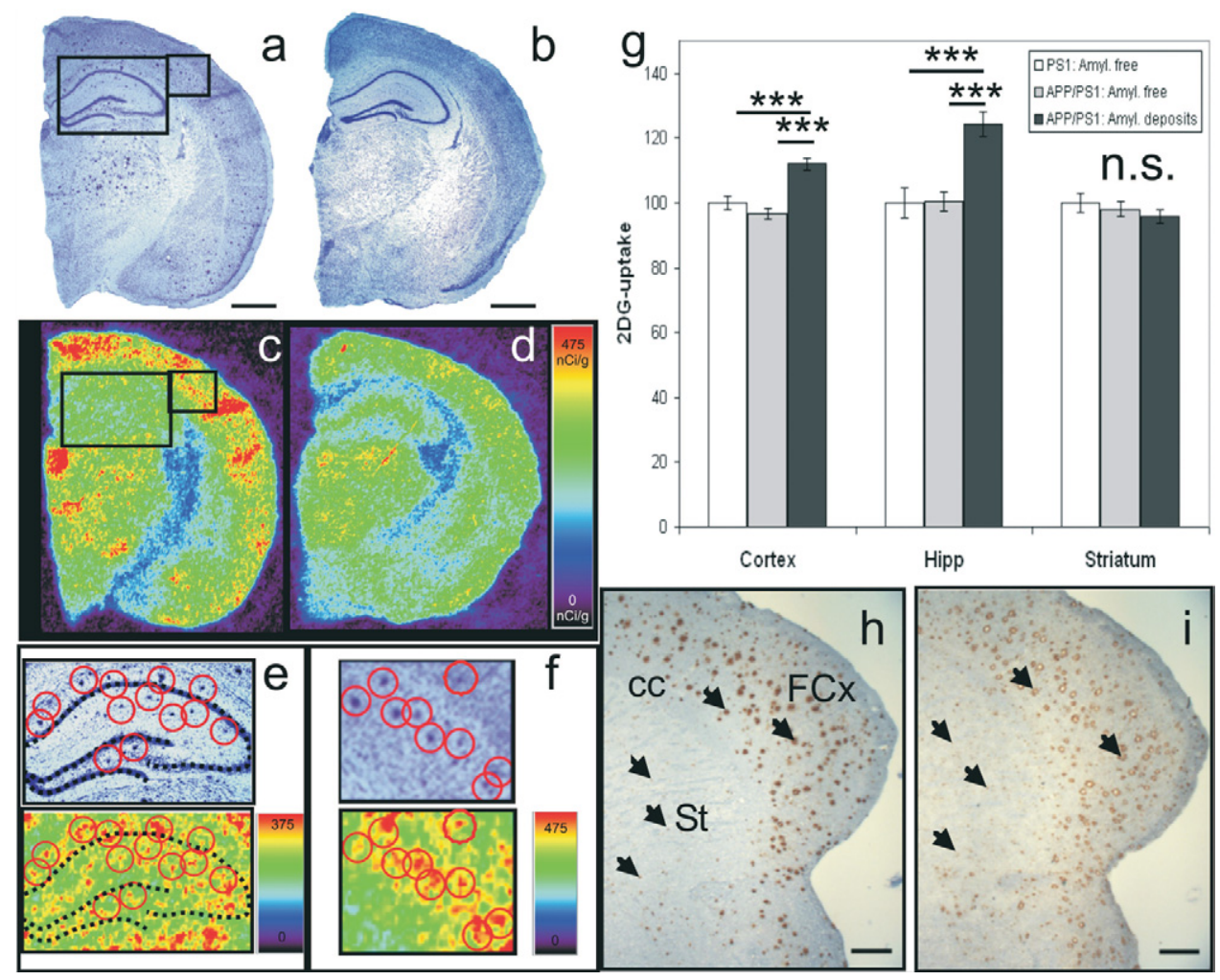

Fig. 5. Evaluation of metabolic activity in brain regions containing amyloid plaques in 12-month-old APP/PS1 mice and control tissue from APP/PS1 and PS1 mice. (a, b) Niss1 stain sections from APP/PS1 (a) and PS1 (b) mice ( $n=3-4$ per group). Insets outlined correspond to magnified Nissl stain sections in (e) and (f). Amyloid plaques were visible on sections from APP/PS1 mice (a; red circles in e and f). Autoradiographic images corresponding to the same sections are shown in (c), (d), (e), and (f). In the autoradiographic section in (e) and (f), an increased glucose uptake is seen within the regions where plaques were present (red circles). (g) Increased glucose uptake is observed in the cortical and hippocampal regions containing amyloid plaques from APP/PS1 mice as compared with amyloid-free regions from APP/PS1 or PS1 mice (analysis of variance [ANOVA] and post hoc analysis, *** $p<0.0001$ ). (h and i) amyloid plaques (h, arrows, 4G8 staining) and activated microglia (I, arrows, Iba1 staining) in the frontal cortex (FCx) and striatum (St) of a 12-month-old APP/PS1 mouse. In the striatum, the density and maturity of amyloid plaques and associated activated microglia were low as compared with those observed in the cortex or hippocampus. Abbreviation: cc, corpus callosum. Autoradiographic scales are in nCi/g. Scale bars, $1 \mathrm{~mm}$ in (a, b); $500 \mu \mathrm{m}$ in (h, i).

an increased cerebral perfusion. Indeed, vascular modifications such as amyloid angiopathy, changed 3-D architecture of brain vasculature, perturbations of the cerbrovascular regulation, leading to altered cerebral blood flow have been shown in AD tg mouse models (Bell and Zlokovic, 2009; Meyer et al., 2008; Niwa et al., 2002). It is thus possible that the higher glucose uptake that we detected by PET was mediated by increased cerebral perfusion linked to vascular alterations. However, our data show that cerebral perfusion is reduced in the cortex of 12-month-old APP/PS1 mice. These results are consistent with data obtained by MRI (Faure et al., 2011) or other methods (Niwa et al., 2002) on other tg mice. Such alteration should reduce and not increase [18F]FDG uptake. We cannot however exclude modifications of the blood brain barrier (BBB) leading to an increased leakage of [18F]-FDG into the brain to explain the increased FDG uptake that we reported. Indeed, such alterations have been reported in tg mouse models of amyloidosis (Ujiie et al., 2003), but not in all (Bourasset et al., 2009).

Second, local modifications occurring in the brain parenchyma or close to the amyloid plaques could also explain increased glucose uptake seen by FDG-PET. In our study, we developed a new protocol to study CGU at the cellular level. Thanks to a spatial normalization algorithm applied to autoradiograms and histological sections, we showed that glucose uptake is increased in regions presenting amyloid plaques as compared with amyloid-free brain regions. Several hypotheses can explain the local CGU increase close to the plaques. For example, the high astrocytosis and microgliosis, that we and others (Bolmont et al., 2008) have seen in association with the plaques, might increase the CGU locally. An increased basal metabolic rate of the cells surrounding amyloid plaques caused by amyloid oligomers (Puzzo et al., 2008), by disturbed astrocytic calcium waves (Kuchibhotla et al., 2009) or by an increased neuronal activity (Busche et al., 2008) might also explain CGU increase close to the plaques. The biological endpoints associated with the increased cerebral glucose uptake in tg mice must be further evaluated.

Several hypothesis can be proposed to explain the decreased [18F]-FDG uptake in the cortex and hippocampus of young 3-month-old APP/PS1 mice. In young mice, the 


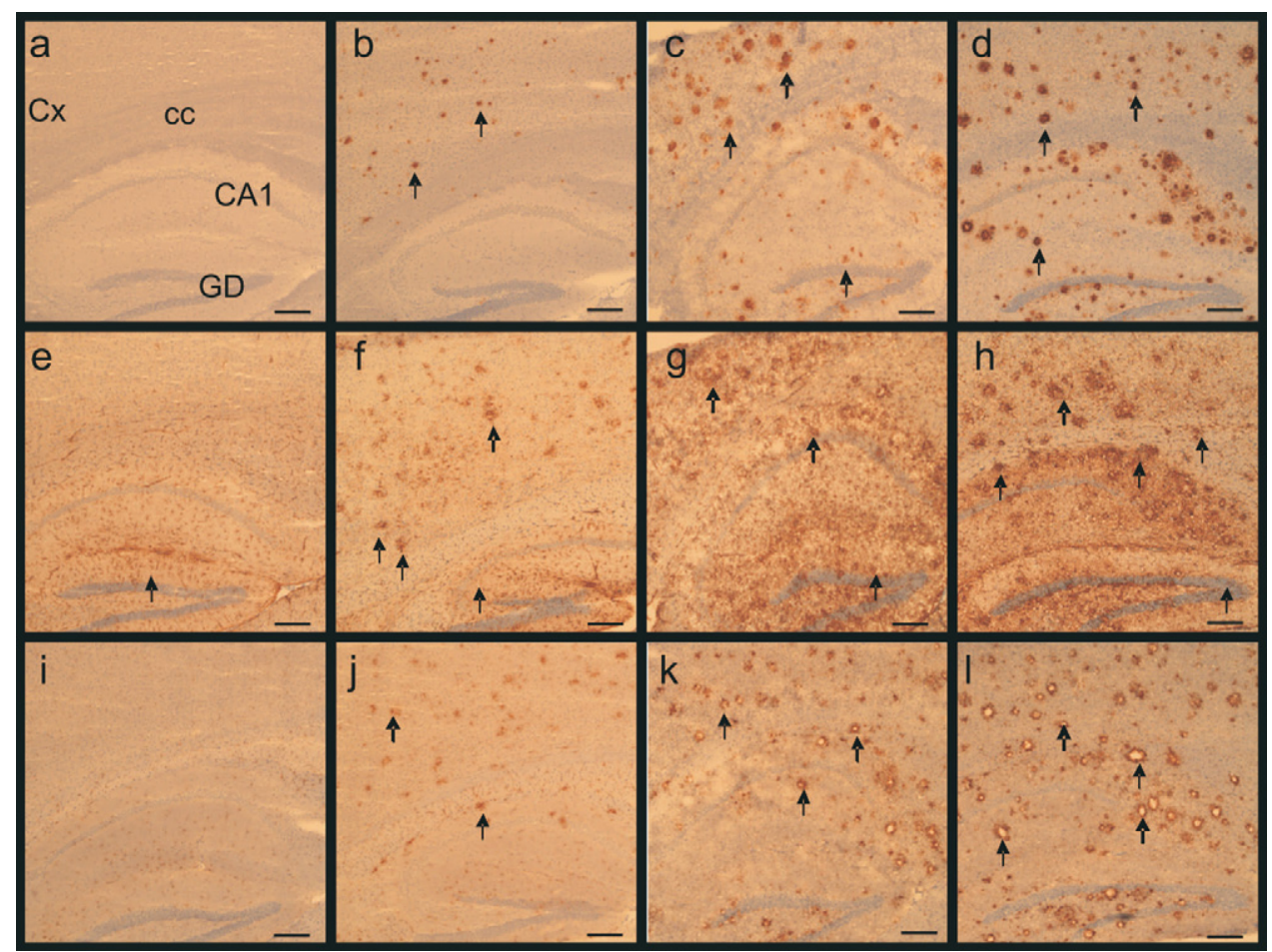

Fig. 6. Increased number and size of amyloid deposits, associated astrocytosis, and microgliosis with aging in APP/PS1 mice. (a-d) Staining for amyloid deposits (arrows) in the cortex and the hippocampus of 3-, 6-, and 12-month-old APP/PS1 mice (b, c, and d, respectively). (a) Lack of staining in a 12-month-old control (C57/B16) mouse (4G8 antibody). (e-h) Reactive astrocytes (arrows) in the brain of a 12-month-old control (C57/B16) mouse (e) and 3- (f), 6- (g), and 12-month-old (h) APP/PS1 mice (glial fibrillary acidic protein [GFAP] antibody). Microgliosis (arrows) in the brain of 3- (j), 6- (k), and 12-month-old (1) APP/PS1 mice (Iba1 antibody). No staining could be detected in control mice (i). Abbreviations: CA1, CA1 region of the hippocampus; cc, corpus callosum; Cx, cortex; DG, dentate gyrus of the hippocampus. Scale bars, $200 \mu \mathrm{m}$.

amyloid plaque load is very low (Blanchard et al., 2003) but other lesions such as intraneuronal $\mathrm{A} \beta$ have been described in this line (Langui et al., 2004). Interestingly it has been evidenced that intraneuronal $\mathrm{A} \beta$ deposition precedes extracellular plaque formation in APP/PS1 mice and that while intracellular $A \beta$ accumulates in young mice it disappears in older animals (Wirths et al., 2001). Intraneuronal A $\beta$ has an exquisite toxicity. In particular, its accumulation leads to synaptic defects and impairments in oxygen consumption (see LaFerla et al., 2007 for review) that could thus lead to a reduced glucose uptake in young APP/PS1 mice. Alternatively it is possible that extracellular soluble $\mathrm{A} \beta$ oligomers leads to the decreased [18F]-FDG uptake in young mice. Oligomers are known to alter synaptic morphology and function (Selkoe, 2008) which in turn can affect brain metabolism. Hence AD patients with no plaques but harboring high levels of $\mathrm{A} \beta$ oligomers undergo severe cerebral hypometabolism (Shimada et al., 2011). A characterization of oligomers deposition in young versus old APP/PS1 mice would provide further information on their association with abnormal glucose uptake in the transgenic mice.

In conclusion, APP/PS1 mice present an age-related increase of cerebral glucose uptake that can be detected at the level of brain regions by in vivo PET imaging and at the level of amyloid plaques by autoradiography. These data suggest a reorganization of glucose uptake in the vicinity of amyloid plaques. Understanding the origin of these changes will help to clarify disease mechanisms associated to $A D$ and to screen potential treatments.

\section{Disclosure statement}

The authors declare no proprietary interest or any conflict of interest related to this study.

The treatment of animals was in accordance with ethical standards of the statutory order $87 / 848$ (October 13, 1987) of the French Ministry of Agriculture (authorization $n^{\circ}$ 91-166).

\section{Acknowledgements}

This work was supported by Medicen (Pôle de compétitivité Ile-de-France, TransAl program), the France Alzheimer association and the NIH (R01-AG020197). We thank Martine Guillermier, Philippe Gervais, Marion Chaigneau, Diane Houitte, Vincent Brulon, Yoann Fontyn, and Pascal Merceron for help with the PET scans, Gilles Bonvento for help with the autoradiographical study, and Fanny Petit for technical help during histological investigations. 


\section{References}

Barrio, J.R., Kepe, V., Satyamurthy, N., Huang, S.C., Small, G., 2008. Amyloid and tau imaging, neuronal losses and function in mild $\operatorname{cog}$ nitive impairment. J. Nutr. Health Aging 12, 61S-65S.

Bell, R.D., Zlokovic, B.V., 2009. Neurovascular mechanisms and bloodbrain barrier disorder in Alzheimer's disease. Acta Neuropathol. 118, $103-113$.

Blanchard, V., Moussaoui, S., Czech, C., Touchet, N., Bonici, B., Planche, M., Canton, T., Jedidi, I., Gohin, M., Wirths, O., Bayer, T.A., Langui, D., Duyckaerts, C., Tremp, G., Pradier, L., 2003. Time sequence of maturation of dystrophic neurites associated with Abeta deposits in APP/PS1 transgenic mice. Exp. Neurol. 184, 247-263.

Bolmont, T., Haiss, F., Eicke, D., Radde, R., Mathis, C.A., Klunk, W.E., Kohsaka, S., Jucker, M., Calhoun, M.E., 2008. Dynamics of the microglial/amyloid interaction indicate a role in plaque maintenance. J. Neurosci. 28, 4283-4292.

Bourasset, F., Mélissa, O., Tremblay, C., Julien, C., Do, T.M., Oddo, S., Laferla, F., Calon, F., 2009. Reduction of the cerebrovascular volume in a transgenic mouse model of Alzheimer's disease. Neuropharmacology $56,808-813$.

Busche, M.A., Eichhoff, G., Adelsberger, H., Abramowski, D., Wiederhold, K.H., Haass, C., Staufenbiel, M., Konnerth, A., Garaschuk, O., 2008. Clusters of hyperactive neurons near amyloid plaques in a mouse model of Alzheimer's disease. Science 321, 1686-1689.

Choo, I.H., Lee, D.Y., Youn, J.C., Jhoo, J.H., Kim, K.W., Lee, D.S., Lee, J.S., Woo, J.I., 2007. Topographic patterns of brain functional impairment progression according to clinical severity staging in 116 Alzheimer disease patients: FDG-PET study. Alzheimer Dis. Assoc. Disord. 21, 77-84.

Cohen, A.D., Price, J.C., Weissfeld, L.A., James, J., Rosario, B.L., Bi, W., Nebes, R.D., Saxton, J.A., Snitz, B.E., Aizenstein, H.A., Wolk, D.A., Dekosky, S.T., Mathis, C.A., Klunk, W.E., 2009. Basal cerebral metabolism may modulate the cognitive effects of Abeta in mild cognitive impairment: an example of brain reserve. J. Neurosci. 29, 1477014778.

Constantinescu, C.C., Mukherjee, J., 2009. Performance evaluation of an Inveon PET preclinical scanner. Phys. Med. Biol. 54, 2885-2899.

Delatour, B., Guégan, M., Volk, A., Dhenain, M., 2006. In vivo MRI and histological evaluation of brain atrophy in APP/PS1 transgenic mice. Neurobiol. Aging 27, 835-847.

Dodart, J.C., Mathis, C., Bales, K.R., Paul, S.M., Ungerer, A., 1999. Early regional cerebral glucose hypometabolism in transgenic mice overexpressing the V717F beta-amyloid precursor protein. Neurosci. Lett. $277,49-52$.

Dubois, A., Dauguet, J., Herard, A.S., Besret, L., Duchesnay, E., Frouin, V., Hantraye, P., Bonvento, G., Delzescaux, T., 2007. Automated three-dimensional analysis of histological and autoradiographic rat brain sections: application to an activation study. J. Cereb. Blood Flow Metab. 27, 1742-1755.

Duyckaerts, C., Potier, M.C., Delatour, B., 2008. Alzheimer disease models and human neuropathology: similarities and differences. Acta Neuropathol. 115, 5-38.

Edison, P., Archer, H.A., Hinz, R., Hammers, A., Pavese, N., Tai, Y.F., Hotton, G., Cutler, D., Fox, N., Kennedy, A., Rossor, M., Brooks, D.J., 2007. Amyloid, hypometabolism, and cognition in Alzheimer disease: an [11C]PIB and [18F]FDG PET study. Neurology 68, 501-508.

El Tannir El Tayara, N., Delatour, B., Le Cudennec, C., Guégan, M., Volk, A., Dhenain, M., 2006. Age-related evolution of amyloid burden, iron load, and MR relaxation times in a transgenic mouse model of Alzheimer's disease. Neurobiol. Dis. 22, 199-208.

Faure, A., Verret, L., Bozon, B., El Tannir El Tayara, N., Ly, M., Kober, F., Dhenain, M., Rampon, C., Delatour, B., 2011. Impaired neurogenesis, neuronal loss, and brain functional deficits in the APPxPS1-Ki mouse model of Alzheimer's disease. Neurobiol. Aging 32, 407-418.
Gonzalez-Lima, F., Berndt, J.D., Valla, J.E., Games, D., Reiman, E.M., 2001. Reduced corpus callosum, fornix and hippocampus in PDAPP transgenic mouse model of Alzheimer's disease. Neuroreport 12, 2375-2379.

Heneka, M.T., Ramanathan, M., Jacobs, A.H., Dumitrescu-Ozimek, L., Bilkei-Gorzo, A., Debeir, T., Sastre, M., Galldiks, N., Zimmer, A., Hoehn, M., Heiss, W.D., Klockgether, T., Staufenbiel, M., 2006. Locus ceruleus degeneration promotes Alzheimer pathogenesis in amyloid precursor protein 23 transgenic mice. J. Neurosci. 26, 1343-1354.

Herholz, K., Carter, S.F., Jones, M., 2007. Positron emission tomography imaging in dementia. Br. J. Radiol. 80(Spec No 2), S160-S167.

Hsiao, K., Chapman, P., Nilsen, S., Eckman, C., Harigaya, Y., Younkin, S., Yang, F., Cole, G., 1996. Correlative memory deficits, Abeta elevation, and amyloid plaques in transgenic mice. Science 274, 99-102.

Jellinger, K.A., Bancher, C., 1998. Neuropathology of Alzheimer's disease: a critical update. J. Neural Transm. Suppl. 54, 77-95.

Kober, F., Duhamel, G., Cozzone, P.J., 2008. Experimental comparison of four FAIR arterial spin labeling techniques for quantification of mouse cerebral blood flow at 4.7 T. NMR Biomed. 21, 781-792.

Kuchibhotla, K.V., Lattarulo, C.R., Hyman, B.T., Bacskai, B.J., 2009. Synchronous hyperactivity and intercellular calcium waves in astrocytes in Alzheimer mice. Science 323, 1211-1215.

Kuntner, C., Kesner, A.L., Bauer, M., Kremslehner, R., Wanek, T., Mandler, M., Karch, R., Stanek, J., Wolf, T., Müller, M., Langer, O., 2009. Limitations of small animal PET imaging with [18F]FDDNP and FDG for quantitative studies in a transgenic mouse model of Alzheimer's disease. Mol. Imaging Biol. 11, 236-240.

LaFerla, F.M., Green, K.N., Oddo, S., 2007. Intracellular amyloid-beta in Alzheimer's disease. Nat. Rev. Neurosci. 8, 499-509.

Langui, D., Girardot, N., El Hachimi, K.H., Allinquant, B., Blanchard, V., Pradier, L., Duyckaerts, C., 2004. Subcellular Topography of Neuronal A $\beta$ Peptide in APPxPS1 Transgenic Mice. Am. J. Pathol. 165, 14651477.

Luo, F., Rustay, N.R., Ebert, U., Hradil, V.P., Cole, T.B., Llano, D.a., Mudd, S.R., Zhang, Y., Fox, G.B., Day, M., 2010. Characterization of 7-and 19-month-old Tg2576 mice using multimodal in vivo imaging: limitations as a translatable model of Alzheimer's disease. Neurobiol. Aging doi:10.1016/j.neurobiolaging.2010.08.005.

Matsumura, A., Mizokawa, S., Tanaka, M., Wada, Y., Nozaki, S., Nakamura, F., Shiomi, S., Ochi, H., Watanabe, Y., 2003. Assessment of microPET performance in analyzing the rat brain under different types of anesthesia: comparison between quantitative data obtained with microPET and ex vivo autoradiography. Neuroimage 20, 2040-2050.

Meyer, E.P., Ulmann-Schuler, A., Staufenbiel, M., Krucker, T., 2008. Altered morphology and 3D architecture of brain vasculature in a mouse model for Alzheimer's disease. Proc. Natl. Acad. Sci. U. S. A. 105, 3587-3592.

Mosconi, L., 2005. Brain glucose metabolism in the early and specific diagnosis of Alzheimer's disease. FDG-PET studies in MCI and AD. Eur. J. Nucl. Med. Mol. Imaging 32, 486-510.

Mosconi, L., Tsui, W.H., De Santi, S., Li, J., Rusinek, H., Convit, A., Li, Y., Boppana, M., de Leon, M.J., 2005. Reduced hippocampal metabolism in MCI and AD: automated FDG-PET image analysis. Neurology 64, 1860-1867.

Nestor, P.J., Scheltens, P., Hodges, J.R., 2004. Advances in the early detection of Alzheimer's disease. Nat. Med. 10, S34-S41.

Nicholson, R.M., Kusne, Y., Nowak, L.A., LaFerla, F.M., Reiman, E.M., Valla, J., 2010. Regional cerebral glucose uptake in the 3xTG model of Alzheimer's disease highlights common regional vulnerability across AD mouse models. Brain Res. 1347, 179-185.

Nicolakakis, N., Aboulkassim, T., Ongali, B., Lecrux, C., Fernandes, P., Rosa-Neto, P., Tong, X.K., Hamel, E., 2008. Complete rescue of cerebrovascular function in aged Alzheimer's disease transgenic mice by antioxidants and pioglitazone, a peroxisome proliferator-activated receptor gamma agonist. J. Neurosci. 28, 9287-9296. 
Niwa, K., Kazama, K., Younkin, S.G., Carlson, G.A., Iadecola, C., 2002. Alterations in cerebral blood flow and glucose utilization in mice overexpressing the amyloid precursor protein. Neurobiol. Dis. 9, 61-68.

Paxinos, G., Franklin, K.B.J., 2001. The Mouse Brain in Stereotaxic Coordinates, second ed. Academic Press, San Diego.

Phelps, M.E., 2006. PET Physics, Instrumentation, and Scanners. New York: Springer Science + Business Media.

Puzzo, D., Privitera, L., Leznik, E., Fa, M., Staniszewski, A., Palmeri, A., Arancio, O., 2008. Picomolar amyloid-beta positively modulates synaptic plasticity and memory in hippocampus. J. Neurosci. 28, 1453714545.

Reiman, E.M., Uecker, A., Gonzalez-Lima, F., Minear, D., Chen, K., Callaway, N.L., Berndt, J.D., Games, D., 2000. Tracking Alzheimer's disease in transgenic mice using fluorodeoxyglucose autoradiography. Neuroreport 11, 987-991.

Selkoe, D.J., 2008. Soluble oligomers of the amyloid beta-protein impair synaptic plasticity and behavior. Behav. Brain Res. 192, 106-113.

Shimada, H., Ataka, S., Tomiyama, T., Takechi, H., Mori, H., Miki, T., 2011. Clinical Course of Patients with Familial Early-Onset Alzheimer's Disease Potentially Lacking Senile Plaques Bearing the E693A Mutation in Amyloid Precursor Protein. Dement. Geriatr. Cogn. Disord. $32,45-54$

Silverman, D.H., Small, G.W., Chang, C.Y., Lu, C.S., Kung De Aburto, M.A., Chen, W., Czernin, J., Rapoport, S.I., Pietrini, P., Alexander, G.E., Schapiro, M.B., Jagust, W.J., Hoffman, J.M., Welsh-Bohmer, K.A., Alavi, A., Clark, C.M., Salmon, E., de Leon, M.J., Mielke, R., Cummings, J.L., Kowell, A.P., Gambhir, S.S., Hoh, C.K., Phelps, M.E., 2001. Positron emission tomography in evaluation of dementia: Regional brain metabolism and long-term outcome. JAMA 286, 2120 2127.

Sun, Y., Schmidt, N.O., Schmidt, K., Doshi, S., Rubin, J.B., Mulkern, R.V., Carroll, R., Ziu, M., Erkmen, K., Poussaint, T.Y., Black, P., Albert, M., Burstein, D., Kieran, M.W., 2004. Perfusion MRI of U87 brain tumors in a mouse model. Magn. Reson. Med. 51, 893-899.
Tai, Y.C., Ruangma, A., Rowland, D., Siegel, S., Newport, D.F., Chow, P.L., Laforest, R., 2005. Performance evaluation of the microPET focus: a third-generation microPET scanner dedicated to animal imaging. J. Nucl. Med. 46, 455-463.

Toyama, H., Ichise, M., Liow, J.S., Modell, K.J., Vines, D.C., Esaki, T., Cook, M., Seidel, J., Sokoloff, L., Green, M.V., Innis, R.B., 2004. Absolute quantification of regional cerebral glucose utilization in mice by 18 F-FDG small animal PET scanning and 2-14C-DG autoradiography. J. Nucl. Med. 45, 1398-1405.

Ujiie, M., Dickstein, D.L., Carlow, D.A., Jefferies, W.A., 2003. Bloodbrain barrier permeability precedes senile plaque formation in an Alzheimer disease model. Microcirculation 10, 463-470.

Valla, J., Gonzalez-Lima, F., Reiman, E.M., 2008. FDG autoradiography reveals developmental and pathological effects of mutant amyloid in PDAPP transgenic mice. Int. J. Dev. Neurosci. 26, 253-258.

Valla, J., Schneider, L., Reiman, E.M., 2006. Age- and transgene-related changes in regional cerebral metabolism in PSAPP mice. Brain Res. 1116, 194-200.

Vlassenko, A.G., Vaishnavi, S.N., Couture, L., Sacco, D., Shannon, B.J., Mach, R.H., Morris, J.C., Raichle, M.E., Mintun, M.A., 2010. Spatial correlation between brain aerobic glycolysis and amyloid- $\beta$ (A $\beta)$ deposition. Proc. Natl. Acad. Sci. U. S. A. 107, 17763-17767.

Williams, D.S., Detre, J.A., Leigh, J.S., Koretsky, A.P., 1992. Magnetic resonance imaging of perfusion using spin inversion of arterial water. Proc. Natl. Acad. Sci. U. S. A. 89, 212-216.

Wirths, O., Multhaup, G., Czech, C., Blanchard, V., Moussaoui, S., Tremp, G., Pradier, L., Beyreuther, K., Bayer, T.A., 2001. Intraneuronal Abeta accumulation precedes plaque formation in beta-amyloid precursor protein and presenilin-1 double-transgenic mice. Neurosci. Lett. 306, $116-120$.

Yu, A.S., Lin, H.D., Huang, S.C., Phelps, M.E., Wu, H.M., 2009. Quantification of cerebral glucose metabolic rate in mice using 18F-FDG and small-animal PET. J. Nucl. Med. 50, 966-973.

Zahs, K.R., Ashe, K.H., 2010. "Too much good news"-are Alzheimer mouse models trying to tell us how to prevent, not cure, Alzheimer's disease? Trends Neurosci. 33, 381-389. 